\title{
High within-host genetic variation of the nematode Spirocerca lupi in a high-density urban dog population
}

\author{
Pamela J. de Waal ${ }^{\mathrm{a}}$, Annemarie Gous ${ }^{\mathrm{a}}$, Sarah J. Clift ${ }^{\mathrm{b}}$ \& Jaco M. Greeff ${ }^{\mathrm{a}, *}$ \\ ${ }^{a}$ Department of Genetics, University of Pretoria, Pretoria 0002, South Africa \\ ${ }^{\mathrm{b}}$ Section Pathology, Department of Paraclinical Sciences, Faculty of Veterinary Science, University of Pretoria \\ *Corresponding author at: Department of Genetics, University of Pretoria, Pretoria 0002, South Africa. Tel.: +27 \\ 12362 5327; fax: +27 123625327. \\ E-mail addresses: jaco.greeff@up.ac.za,Pam.deWaal@up.ac.za, sarah.clift@up.ac.za; \\ annemarie.gous@gmail.com
}

\begin{abstract}
The nematode worm Spirocerca lupi has a cosmopolitan distribution and can cause the death of its final canid host, typically dogs. While its life cycle, which involves a coprophagous beetle intermediate host, a number of non-obligatory vertebrate paratenic hosts and a canid final host, is well understood, surprisingly little is known about its transmission dynamics and population genetic structure. Here we sequenced cox 1 to quantify genetic variation and the factors that limit gene flow in a $300 \mathrm{~km}^{2}$ area in South Africa. Three quarters of the genetic variation, was explained by differences between worms from the same host, whereas a quarter of the variation was explained by differences between worms from different hosts. With the help of a newly derived model we conclude that while the offspring from different infrapopulations mixes fairly frequently in new hosts, the level of admixture is not enough to homogenize the parasite populations among dogs. Small infrapopulation sizes along with clumped transmission may also result in members of infrapopulations being closely related.
\end{abstract}

Keywords: parasite, canid, population genetic structure, nematode, transmission 


\section{Introduction}

Spirocerca lupi occurs globally in tropical and sub-tropical areas and causes spirocercosis in canids, predominantly dogs (van der Merwe et al., 2008). Spirocercosis can be fatal when the oesophageal nodules undergo malignant transformation to sarcoma, with subsequent metastasis to other sites (van der Merwe et al., 2008). Spirocercosis is also fatal when it causes rupture of the aorta during larval migration. Occasionally, aberrant larval migration to inappropriate tissues can result in a variety of life threatening problems (Dvir et al., 2010). In recent years South African and Israeli veterinarians have reported an increase in spirocercosis (van der Merwe et al., 2008).

Spirocerca lupi's life cycle can be summarised as follows: eggs containing first stage larvae (L1) are ingested by coprophagous beetles where the worm develops to the third stage larvae (L3). If an infected beetle is consumed by a paratenic host, which include birds, lizards and rodents, the larvae excyst and then re-encyst in a variety of tissues (Bailey, 1972; van der Merwe et al., 2008). The final host can contract spirocercosis by consuming either the beetle or the paratenic host. Ingested L3 larvae penetrate the gastric mucosa and migrate in the wall of the gastric and coeliac arteries to the caudal thoracic aorta, where they moult to L4 and finally to adults. Young adult worms then migrate from the aorta to the oesophagus below, where they induce the formation of nodules. From here they lay eggs into the dog's alimentary tract.

All quantitative studies suggest that $S$. lupi populations undergo frequent fluxes (Chandrasekharon et al., 1958; Bailey, 1972) and the recent increases may be just that, or it could also be the result of increased vigilance of veterinarians for the parasite. Traversa et al. (2007) genotyped 20 S. lupi worms originating from Africa, Asia and Europe and found that their samples from Israel (7), Iran (3) and South Africa (4) were identical. Given the vast distances, this observation may suggest a very recent global expansion of one mitochondrial DNA haplotype, possibly explaining the observed increase in the incidence of cases.

Similar to some macroparasites (Nadler, 1995; Criscione et al., 2010; Ferguson et al., 2011), S. lupi has fairly small infrapopulation sizes - based on counts in du Toit et al. (2008) and van der Merwe et al. (2008) a minimum estimate of the harmonic mean infrapopulation size is $N=6$ (the harmonic mean number reflects the effective population size the best; Nadler, 1995; Hedrick, 2000). In such a small population, genetic drift will reduce genetic variation of the population of co-transmitted parasites, while increasing the genetic differences between populations of co-transmitted parasites (Nadler, 1995; Sire et al., 2001; 
Criscione and Blouin, 2006; Steinauer et al., 2010). For instance, if two alleles are present at equal frequencies of 0.5 , one of the alleles is expected to be lost within $2.8 N$ generations (Hedrick, 2000). In other words, if subpopulations of parasites do not merge and cycle between the final and intermediate host, genetic variation within the subpopulation will be lost in $2.8 \times 6 / 2=8.4$ generations. The division by two is because there is a sampling effect going from the dog to the beetle and from the beetle to the dog. For mitochondrial variation this clearance of genetic variation will occur even faster because only females transmit the DNA and have only one copy each, i.e. if the sex ratio is 50:50 it will clear four times faster and the subpopulation will be homogenous at their mitochondria within 2 life cycles.

On the other hand, the merger of subpopulations of parasite into one infrapopulation results in gene flow that will homogenize the genetic variation between infrapopulations (the parasites of a specific species in one host (Margolis et al., 1982)). Such mergers can occur when third stage larvae from different intermediate/paratenic hosts are consumed by one paratenic/final host or when an intermediate host consumes faeces from different infected dogs resulting in the merger of the descendants of different infrapopulations. Therefore we can deduce the rate of amalgamation of genes from different infrapopulations by determining how genetic variation is structured within and between hosts (Criscione and Blouin, 2006; Archie et al., 2008; Steinauer et al., 2010).

Although studies on parasitic helminths have found situations where there is no genetic distinction between infrapopulations (Criscione and Blouin, 2006; Johnson et al. 2006) it is more common to have some discernable genetic differences between infrapopulations although these differences tend to be small (Mulvey et al., 1991; Anderson et al., 1995; Jobet et al., 2000; Sire et al., 2001; Criscione et al., 2007). Significant between-host differences are frequently explained as a result of clumped transmission and small infrapopulation sizes resulting in little gene flow and strong drift (Nadler, 1995; Criscione et al., 2010), whereas small differences are associated with larger infrapopulations (Sire et al., 2001; Theron et al., 2004; Johnson et al., 2006). Irrespective of the between host differences, it is possible to find no genetic structure over vast geographic distances (Jefferies et al., 2010; Baldwin et al., 2011) or significant structure (Wu et al., 2009; Belanger et al., 2011), in some populations, even at a local scale (Criscione et al., 2010).

Nothing is known about the population genetic structure and transmission dynamics of S. lupi. The aim of this study is therefore to quantify how genetic variation of $S$. lupi is structured within and between hosts in order to gain an appreciation of transmission 
dynamics. To this end we develop a model for relating the rate of population amalgamation with $F_{S T}$. We can also use the genetic variation to test if the population size has been growing.

\section{Materials and methods}

\subsection{Spirocerca samples and DNA extraction}

DNA was isolated from three worms per dog for twenty dogs giving a total sample size of $60 S$. lupi individuals. These worms were collected at the Onderstepoort Veterinary Academic Hospital of the University of Pretoria (the largest referral hospital in Africa). The samples were obtained from dogs brought to the facility for necropsy. All dogs from which $S$. lupi individuals were sampled originated from the Pretoria and Centurion areas in South Africa (Fig. 1).

All worms used for DNA extraction were individually washed three times with $96 \%$ ethanol prior to transfer to separate Eppendorf tubes. The worms were crushed using liquid nitrogen. DNA was extracted using the DNeasy® Blood and Tissue Kit (Qiagen) following the manufacturer's protocol for DNA extraction from insects. All samples were lysed overnight at $55^{\circ} \mathrm{C}$ before completion of the procedure.

\subsection{PCR amplification and sequencing}

Genetic variation was quantified by sequencing a mitochondrial gene, cytochrome oxidase I ( $\operatorname{cox} 1)$. The $\operatorname{cox} 1$ mitochondrial gene has a high evolutionary rate (Powers, 2004), which makes the gene a good candidate for the study of intraspecific genetic variation. In the study of parasitic nematodes, coxl has been used to characterize genetic variation within and between species (Hu et al., 2002; Hebert et al., 2003; Otranto et al., 2005).

Spirocerca lupi coxl was amplified using primers previously designed and successfully used in nematodes (Bowles et al., 1992; Hu et al., 2002), namely JB3 (5' TTTTTTGGGCATCCTGAGGTTTAT 3') and JB4.5 (5' AAAGAAAGAACATAATGAAAATG 3'). These primers yielded an amplicon of approximately $440 \mathrm{bp}$. PCR reactions of $50 \mu 1$ were performed using approximately $100 \mathrm{ng}$ of S. lupi DNA, 25 pmol of each primer, $10 \mathrm{mM}$ of each dNTP, $3 \mathrm{mM} \mathrm{MgCl}_{2}, 0.25 \mathrm{U}$ AmpliTaq Gold (Applied Biosystems) and GeneAmp PCR Gold Buffer to a final concentration of 15 $\mathrm{mM}$ Tris- $\mathrm{HCl}, 50 \mathrm{mM} \mathrm{KCl}$ ( $\mathrm{pH} 8.0$ at room temperature). The PCR reaction conditions were 
adapted from a study of ITS in Thelazia (Otranto et al., 2001). The programme consisted of an initial denaturation of DNA and activation of polymerase step of 5 minutes at $95^{\circ} \mathrm{C}$ followed by 35 cycles of $95^{\circ} \mathrm{C}$ for 1 minute, $58^{\circ} \mathrm{C}$ for 1 minute and $72^{\circ} \mathrm{C}$ for 1 minute. A final elongation step of 5 minutes at $72^{\circ} \mathrm{C}$ completed the amplification process (Eppendorf Gradient Mastercycler). PCR products were purified using the High Pure PCR Product Purification Kit (Roche Diagnostics).

Genomic DNA was used as a template for the amplification of a 440bp cox 1 fragment. Amplicons were sent to Macrogen Inc, Rockville, USA, where sequencing was performed using an ABI 3770 automated sequencer. Sequences were viewed and aligned in BioEdit (Hall, 1999) using the Clustal W algorithm (Thompson et al., 1994). Manual analysis of sequences was performed to ensure complete accuracy.

\subsection{Statistical analyses}

An AMOVA was performed in Arlequin 3.11 (Excoffier et al., 2005) to test two levels of population subdivision: 1) the dog host from which worms came (i.e. the infrapopulations) and 2) the north and south sides of the Magaliesberg (mountain, Fig. 1). A Mantel test was used to correlate the genetic and geographic distances between infrapopulations. Significance was estimated with 10000 permutations (Excoffier et al. 1992). The overall $\varphi_{\text {ST }}$ between infrapopulations was also estimated (Weir and Cockerham, 1984).

To obtain a visual appreciation for how clumped genotypes were in dog hosts, a null distribution of the number of haplotypes per dog was created by assigning the observed haplotypes randomly to each host ( $10^{6}$ times). This distribution can be visualised in a ternary plot with axes for the number of dogs with one worm haplotype, two worm haplotypes and three worm haplotypes respectively. Significance was calculated as the sum of the probability of the observed case and all more extreme cases (these calculations were performed in $\mathrm{R}$ version 2.11.0 (R Development Core Team, 2010)).

Gene genealogies were calculated with statistical parsimony (Tempelton et al., 1992) as implemented in TCS version 1.21 (Clement et al., 2000). The gene network showed two haplogroups which may be the result of two former populations merging. To test this idea Chakraborty's test of population amalgamation (Chakraborty, 1990) as implemented in Arlequin 3.11 (Excoffier et al., 2005) was run on the data. For this test (and the next) we trimmed our data by deleting repeated samples from the same dog as this can be the result of recent ancestry so that the data cannot be considered independent (however, runs where all 
haplotypes were included lead to similar conclusions). To test if the population has been expanding the mismatch distribution was analyzed with Arlequin 3.11 (Excoffier et al., 2005), testing if the haplotype distribution differs from an expanding population model (Schneider and Excoffier, 1999) and calculating the raggedness index (Harpending, 1994).

\subsection{Amalgamation model}

From our estimate of $\varphi_{\mathrm{ST}}$, a crude estimate of the number of female migrants can be calculated as $\mathrm{M}=\left(1-\varphi_{\mathrm{ST}}\right) / 2 \varphi_{\mathrm{ST}}$ (Slatkin, 1991). However, Whitlock and McCauley (1999) have argued that these estimates are so dependent on unrealistic assumptions that it is essentially meaningless (Holsinger and Weir, 2009). Here we briefly develop an alternative model that does not make as many restrictive assumptions as the above estimate and takes into account that a fraction of populations merge rather than a constant level of migration, $\mathrm{M}$, between populations. We assume a metapopulation composed of infinitely many subpopulations (in this case each infrapopulation), each containing an effective copy number of $n$ alleles per population. All infrapopulations survive one generation only and a fraction (1 $-a$ ) of the new generation $(t+1)$ populations are formed by sampling at random from a randomly selected infrapopulation from generation $t$. The remaining fraction $a$, of new populations are composed of a 50:50 amalgamation of two infrapopulations, both sampled at random from generation $t . F_{S T}=\left(H_{T}-H_{S}\right) / H_{T}$, with $H_{T}$ equal to the probability that two alleles sampled at random from the entire population are not identical by recent descent and $H_{S}$ is the equivalent probability sampling from a subpopulation only, in our case the infrapopulation. Given that the population is infinite in size $H_{T}$ is normally assumed to be 1 . This means that $F_{S T}=1-H_{S}$ which is equal to the chance that two randomly sampled alleles from a population are identical by recent descent, $f$. We proceed to calculate the equilibrium value of $f$ by setting up a recursion equation: the chances that two randomly drawn alleles from a subpopulation (in our case infrapopulation) are copies of the same gene in the previous generation is $1 / n$ (irrespective of if the new population is an amalgamation or not). Additionally, if the two drawn alleles are not copies from the same allele in generation $t(=1-1 / \mathrm{n}$ in non-amalgamated populations and $=1 / 2-1 / \mathrm{n}$ in amalgamated populations), they may still be identical by recent descent from previous generations and this is equal to $f_{\mathrm{t}-1}$. This yields the recursion equation:

$$
f_{t}=\frac{1}{n}+(1-a)\left(1-\frac{1}{n}\right) f_{t-1}+a\left(\frac{1}{2}-\frac{1}{n}\right) f_{t-1},
$$


setting $f_{t}=f_{t-1}=\widetilde{f}$, and solving for $\widetilde{f}$ gives the equilibrium as:

$\widetilde{f}=\frac{2}{2+n a}$

Seeing as $F_{S T}=\widetilde{f}$, we can rearrange equation (1), inserting our estimates of $F_{S T}$ and $n$ to solve for the rate of amalgamation, $a=2\left(1-F_{S T}\right) / n F_{S T}$. We can use this model to draw $\mathrm{F}_{\mathrm{ST}}$ isoclines in the parameter space of infrapopulation size $(n)$ and the rate of population amalgamation (a) (Fig. 2). Simulations indicate that these equilibria of $F_{S T}$ are reached rapidly (within ten generations).

\section{Results}

\subsection{Population genetic structure}

Eleven haplotypes were identified and their sequences submitted to Genbank under accession numbers HQ674751 to HQ674761 (Table 1). These all translated into amino acids without any stop codons. Within the 346 scored nucleotide sites there were 20 segregating sites, with 18 transitions and 3 transversions. On average there were 6.57 pairwise differences between all the combined sequences, giving a nucleotide diversity $(\pi)$ of 0.01898 . The AMOVA showed that most of the genetic variation, $76 \%$, is explained by differences between worms from the same dog, while a not insignificant fraction $(28 \%)$ of the variation is explained by differences between infrapopulations (Table 2). The sides of the mountain explained none of the genetic variation; in fact the sides were more similar than expected from chance (Table 2). The global $\varphi_{\text {sT }}$ among dogs of 0.272 was significant $(p=0.0086)$ while the pair-wise $\varphi_{\text {ST }}$ values were not correlated with the distances between dogs $(r=$ 0.047; $p=0.658)$.

The random assignment of data underscores this finding showing that haplotypes are significantly more clumped than random ( $p=0.0328$; Fig. 3). Under a random assortment of haplotypes the most likely observation would have been that two of the single haplotype dogs and two of the two-haplotype dogs contained three haplotypes. We note that dogs containing 
a mixture of the two haplotype groups (see below) are nearly significantly underrepresented (chi-squared test: $\mathrm{X}_{2}^{2}=4.218, p=0.06$ ).

At a $95 \%$ connection limit of parsimony, TCS split the coxl network into two, but at $94 \%$ the limit of parsimony was 8 mutation steps, which allowed the two haplotype groups to be connected (Fig. 4). The first haplotype group is comprised of haplotypes 1, 2, 3, 6, 9 and 10, and the second group includes haplotypes 4, 5, 7, 8 and 11 .

\subsection{Population dynamics}

Although the observed number of 11 alleles are more than the expected 8.15, this difference was not significant $(p=0.14)$. The goodness of fit tests show that the estimated expansion model differed significantly from the data $(\mathrm{SSD}=0.1207, p=0.014)$. The raggedness index of 0.279 was significantly greater than the estimated model $(p=0.0001)$ and the mismatch distribution shows the peakiness of a typical stationary population (Fig. 5).

\section{Discussion}

Three quarters of the genetic variation was explained by differences between worms sharing the same host while the remaining quarter was explained by differences between infrapopulations. Although we found two haplogroups of worm, we found no significant evidence to suggest that it is the result of two populations having made secondary contact. We found evidence that the population is stable in size rather than that it has grown. Given the structuring of the genetic variation over infrapopulations and the small infrapopulation sizes, at least $20 \%$ of populations are the result of the amalgamation of offspring originating from different parent infrapopulations, but this value may be as high as $60 \%$.

For coxl we identified 11 haplotypes from sixty sequences in $300 \mathrm{~km}^{2}$. This is more than the 2 haplotypes identified by Traversa et al. (2007) looking at $20 \mathrm{~S}$. lupi worms, but originating from three continents (Africa, Asia and Europe). This low variation of Traversa et al. (2007) is at odds with the high variation we observed at a local scale and more data will be required to resolve this inconsistency.

Neither the AMOVA, nor the Mantel test found any evidence for geographical structure of the studied population. This means that either the winged intermediate beetle hosts, or the final dog hosts move around sufficiently to prevent the build-up of local variants at this geographical scale. Despite the inability of many macroparasites to disperse actively 
their populations are frequently unstructured due to the movement of intermediate or final hosts (Jefferies et al., 2010; Baldwin et al., 2011). More variable DNA markers such as microsatellites may mutate fast enough to pick up local structure, but the coxl data suggest that geographically, S. lupi is unstructured in this population of $300 \mathrm{~km}^{2}$. From a metapopulation perspective it suggests that gene flow is equally likely between all subpopulations.

Even though the $S$. lupi population is unstructured at a local geographical scale, it does not mean that infrapopulations are randomly composed of genotypes from the suprapopulation (Anderson et al. 1995). Our estimate of $\varphi_{\mathrm{ST}}$ in S.lupi is higher than typical estimates for other macroparasitic worms (Table 3) suggesting lower levels of gene flow. However, note that $n$ for mitochondrial DNA is four times smaller than for diploid genomic DNA. The heavy lines in Fig. 2 connects observed mt DNA $F_{S T}$ values with expected $F_{S T}$ values for nuclear DNA. It is clear that these expected values are comparable to estimates of other macroparasites listed in Table 3. Our model suggests that the amount of admixture required to obtain these values of $F_{S T}$ are fairly high: for infrapopulations composed of an average of 24,12 or 6 female worms, respectively 22,45 or $90 \%$ of infrapopulations are a mixture of worms descending from different parent infrapopulations. Even at the lowest of these rates, only $30 \%$ of populations can expect to avoid amalgamation for more than 5 generations. Because we worked with mitochondrial DNA we can only estimate the movement of females, but since this parasite has no free living phase and the sex ratio seems to be unbiased (Anantaraman and Sen 1966), it is hard to imagine how male worms would migrate differently from females. Given that there was no geographical structure, the model assumption that founders are sampled from random parent infrapopulations is not violated at a local scale.

From ecological data gleaned from the literature we can also try to see if subpopulations amalgamate: If we consider that dogs frequently have around 4 nodules, each containing about 6 worms (van der Merwe et al., 2008), and that less than half the consumed larvae normally manage to establish (Bailey, 1972) this gives us a rough estimate of 48 consumed larvae. Although infected beetles can harbour in excess of 100 larvae, the average number in Pretoria is $32(\mathrm{n}=10$, du Toit et al., 2008). This suggests that dogs may have to eat more than one infected beetle to reach the observed infrapopulation size. Population mergers can also occur in paratenic hosts (Rauch et al., 2005; Dávidová et al., 2011) such as lizards or birds that eat intermediate beetle hosts more readily than dogs do and that can be riddled with larvae (Anantaraman and Sen 1966; Bailey, 1972). 
The $\varphi_{\text {ST }}$ between infrapopulations in our urban sample may be uncharacteristically low as compared to rural and wild areas. The urban areas of Pretoria have a 5 times higher prevalence of $S$. lupi larvae in intermediate beetle hosts than surrounding rural areas $(13.5 \%$ versus 2.4\%; du Toit et al., 2008). This high prevalence may be explained by the dense final host populations in urban areas (JMG counted 41 dogs on his block, which is $0.023 \mathrm{~km}^{2}$ giving a density of 1775 dogs per squared kilometre). With such a high prevalence in the intermediate host, final hosts can be expected to be infected by multiple beetles more frequently which will increase the genetic variation of infrapopulations and decrease the differences between infrapopulations (Eppert et al. 2002). In rural settings dog densities are much lower and their distribution is more clumped around farm homesteads. Under natural conditions canids are normally territorial leading to lower densities (Pitt et al., 2003) and our results should thus not be extrapolated to wild populations.

If within host variation is an important determinant of virulence as suggested by theory (Frank, 1996; Berens et al., 2007; Restif, 2009), the more frequent amalgamation of infrapopulations (Eppert et al., 2002) in urban areas should lead to a more virulent parasite population. It may be interesting to compare virulence between such populations.

While our results support the idea that infrapopulations may frequently amalgamate, the small size of the infrapopulations results in sampled worms often being close relatives and in high drift reducing genetic variation within the infrapopulations (Criscione et al., 2005). For this reason $25 \%$ of genetic variation exists between infrapopulations.

At least one long branch can be expected in networks of sampled alleles as the average coalescence time of the last two alleles in a population takes just as long as the coalescence of all the other alleles combined (Hedrick, 2000). A long branch could also result if two formerly isolated parasite populations make secondary contact such as may have occurred with the introduction of European breeds of dog to southern Africa. Although there is one relatively long branch in the network, Chakraborty's amalgamation test showed that there is no evidence that it resulted from underlying population substructure. Tests to see if the perceived increase in spirocercosis could be the result of an increase in the parasite population size suggested that rather than growing, $S$. lupi seems to have been a stable population for a long time. 


\section{Acknowledgements}

We thank members of the Pathology Section for collecting worms and the NRF and the University of Pretoria for the monetary support. AG was funded by the National Research Foundation (NRF) of South Africa grant GUN 2072974 to JMG. Any opinion, findings and conclusions or recommendations expressed in this material are those of the authors and do not necessarily reflect the views of the NRF. Running costs were covered by JMG and a Research Development grant from the University of Pretoria to PdW. Thanks to Carl Roux for pointing out a mistake in our derivation. The authors appreciate the comments of the reviewers.

\section{Conflict of interest}

The authors have no conflict of interest to declare.

\section{References}

Anantaraman, M., Sen, K., 1966. Experimental Spirocercosis in dogs with larvae from paratenic host, Calotes versocolor, the common garden lizard in Madras. J. Parasitol. $52,911-912$.

Anderson, T.J.C., Romero-Abal, M.E., Jaenike, J., 1995. Mitochondrial DNA and Ascaris microepidemiology: the composition of parasite populations from individual hosts, families and villages. Parasitol. 110, 221-229.

Archie, E.A., Luikart, G., Ezenwa, V.O., 2008. Infecting epidemiology with genetics: a new frontier in disease ecology. Trends Ecol. Evol. 24, 21-30.

Bailey, W.S., 1972. Spirocerca lupi: a continuing inquiry. J. Parasitol. 58, 3-22.

Baldwin, R.E., Rew, M.B., Johansson, M.L., Banks, M.A., Jocobson, K.C., 2011. Population structure of three species of Anisakis nematodes recovered from pacific sardines (Sardinops sagax) distributed throughout the California current system. J. Parasitol. 97, 545-554.

Belanger, D.H., Perkins, S.L., Rockwell, R.F., 2011. Inference of population structure and patterns of gene flow in canine heartworm (Dirofilaria immitis) J. Parasitol. 97, 602609. 
Berens, S.J., Brayton, K.A., McElwain, T.F., 2007. Coinfection with antigenically and genetically distinct virulent strains of Babesia bovis is maintained through all phases of the parasite life cycle. Infect. Immun. 75, 5769-5776.

Bowles, J., Blair, D., McManus, D.P., 1992. Genetic variants within the genus Echinococcus identified by mitochondrial DNA sequencing. Molec. Biochem. Parasitol. 54, 165174.

Chakraborty, R., 1990. Mitochondrial DNA polymorphism reveals hidden heterogeneity within some Asian populations. Am. J. Hum. Genet. 47, 87-94.

Chandrasekharon, K.P., Sastry, G.A., Menon, M.N., 1958. Canine spirocercosis with special reference to the incidence and lesions. Brit. Vet. J. 114, 388-395.

Clement, M., Posada, D., Crandall, K.A., 2000. TCS: a computer program to estimate gene genealogies. Molec. Ecol. 9, 1657-1660.

Criscione, C.D., Anderson, J.D., Raby, K., Sudimack, D., Subedi, J., Rai, D.R., Upadhayay, R.P., Jha, B., Williams-Blangero, S., Anderson, T.J.C., 2007. Microsatellite markers for the human nematode parasite Ascaris lumbricoides: development and assessment of utility. J. Parasitol. 93, 704-708.

Criscione, C.D., Anderson, J.D., Sudimack, D., Subedi, J., Upadhayay, R.P., Jha, B., Williams, K.D., Williams-Blangero, S., Anderson, T.J.C., 2010. Landscape genetics reveals focal transmission of a human macroparasite. PLoS Negl. Trop. Dis. 4, e665, $1-8$.

Criscione, C.D., Blouin, M.S., 2006. Minimal selfing, few clones, and no among-host genetic structure in a hermaphroditic parasite with asexual larval propagation. Evolution 60, $553-562$.

Criscione, C.D., Poulin, R., Bluoin, M.S., 2005. Molecular ecology of parasites: elucidating ecological and microevolutionary processes. Molec. Ecol. 14, 2247-2257.

Dávidová, M., Blažek, R., Trichkova, T., Koutrakis, E., Gaygusuz, Ö., Ercan, E., Ondračová, M., 2011. The role of the European bitterling (Rhodeus amarus, Cyprinidae) in parasite accumulation and transmission in riverine ecosystems. Aquat. Ecol. 45, 377387. 
Du Toit, C.A., Scholtz, C.H., Hyman, W.B., 2008. Prevalence of the dog nematode Spirocerca lupi in populations of its intermediate dung beetle host in the Tshwane (Pretoria) Metropole, South Africa. Onderstepoort J. Vet. Res. 75, 315-321.

Dvir, E., Clift, S.J., van der Merwe, L.L., 2010. Review: challenges in diagnosis and treatment of canine spirocercosis. Isr. J. Vet. Med. 65, 5-9.

Eppert, A., Lewis, F.A., Grzywacz, C., Coura-Filhott, P., Caldast, I., Minchella, D.J., 2002. Distribution of Schistosome infections in molluscan hosts at different levels of parasite prevalence. J. Parasitol. 88, 232-236.

Excoffier, L., Laval, G., Schneider, S., 2005. Arlequin ver. 3.0: an integrated software package for population genetics data analysis. Evol. Bioinf. Online 1, 47-50.

Excoffier, L., Smouse, P., Quattro, J., 1992. Analysis of molecular variance inferred from metric distances among DNA haplotypes: Application to human mitochondrial DNA restriction data. Genetics 131, 479-491.

Ferguson, J.A., Woodberry, K., Gillin, C.M., Jackson, D.H., Sanders, J.L., Madigan, W., Bildfell, R.J., Kent, M.L., 2011. Cylicospirura species (nematoda: Spirocercidae) and stomach nodules in cougars (Puma concolor) and bobcats (Lynx rufus) in Oregon. J. Wildlife Dis. 47, 140-153.

Frank, S.A., 1996. Models of parasite virulence. Q. Rev. Biol. 71, 37-78.

Hall, T.A., 1999. BioEdit: a user-friendly biological sequence alignment editor and analysis program for windows 95/98/NT. Nucl. A. Symp. Series 41, 95-98.

Harpending, R.C., 1994. Signature of ancient population growth in a low-resolution mitochondrial DNA mismatch distribution. Hum. Biol. 66, 591-600.

Hebert, P.D.N., Ratnashingham, S., deWaard, J.R., 2003. Barcoding animal life: cytochrome c oxidase subunit 1 divergence among closely related species. Proc. R. Soc. Lond. B 270, S96-S99.

Hedrick, P.W., 2000. Genetics of Populations. $2^{\text {nd }}$ ed., Jones and Bartlett Publishers, Inc., Sudbury.

Holsinger, K.E., Weir, B.S., 2009. Genetics in geographically structured populations: defining, estimating and interpreting $F_{\mathrm{ST}}$. Nat. Rev. Genet. 10, 639-650. 
Hu, M., Chilton, N.B., Zhu, X., Gasser, R.B., 2002. Single-strand conformation polymorphism-based analysis of mitochondrial cytochrome c oxidase subunit 1 reveals significant substructuring in hookworm populations. Electrophoresis 23, 2734.

Jefferies, R., Shaw, S.E., Willesen, J., Viney, M.E., Morgan, E.R., 2010. Elucidating the spread of the emerging canid nematode Angiostrongylus vasorum between Palaearctic and Nearctic ecozones. Inf. Genet. Evol. 10, 561-568.

Jobet, E., Durand, P., Langand, J., Müller-Graf, C.D.M., Hugot, J.-P., Bougnoux, M.-E., Rivault, C., Cloarec, A., Morand, S., 2000. Comparative genetic diversity of parasites and their hosts: population structure of an urban cockroach and its haplodiploid parasite (oxyuroid nematode). Molec. Ecol. 9, 481-486.

Johnson, P.C.D., Webster, L.M.I., Adam, A., Buckland, R., Dawson, D.A., Keller, L.F., 2006. Abundant variation in microsatellites of the parasitic nematode Trichostrongylus tenius and linkage to tandem repeat. Molec. Biochem. Parasitol. 148, 210-218.

Margolis, L., Esch, G.W., Holmes, J.C., Kuris, A.M., Schad, G.A., 1982. The use of ecological terms in parasitology (report of an ad hoc committee of the American Society of Parasitologists) J. Parasitol. 68, 131-133.

Mulvey, M., Aho, J.M., Lydeard, C., Leberg, P.L., Smith, M.H., 1991. Comparative population genetic structure of a parasite (Fascioloides magna) and its definitive host. Evolution 45, 1628-1640.

Nadler, S.A., 1995. Microevolution and the genetic structure of parasite populations. J. Parasitol. 81, 395-403.

Nadler, S.A., Lindquist, R.L., Near, T.J., 1995. Genetic structure of midwestern Ascars suum populations: a comparison of isozyme and RAPD markers. J. Parasitol. 81, 385-394.

Otranto, D., Tarsitano, E., Traversa, D., Giangaspero, A., De Luca, F., Puccini, V., 2001. Differentiation among three species of bovine Thelazia (Nematoda: Thelaziidae) by polymerase chain reaction-restriction fragment length polymorphism of the first internal transcribed spacer ITS-1 (rDNA). Int. J. Parasitol. 31, 1693-1698.

Otranto, D., Testini, G. de Luca, F., Hu, M., Shamsi, S., Gasser, R.B., 2005. Analysis of genetic variability within Thelazia callipaeda (Nematoda: Thelazioidea) from Europe 
and Asia by sequencing and mutation scanning of the mitochondrial cytochrome $\mathrm{c}$ oxidase subunit 1 gene. Molec. Cel. Probes 19, 306-313.

Pitt, W.C., Box, P.W., Knowlton, F.F., 2003. An individual-based model of canid populations: modelling territoriality and social structure. Ecol. Model. 166, 109-121.

Powers, T., 2004. Nematode molecular diagnostics: from bands to barcodes. Ann. Rev. Phytopathol. 42, 367-383.

R Development Core Team, 2010. R: A language and environment for statistical computing. R Foundation for Statistical Computing, Vienna, Austria.

Rauch, G., Kalbe, M., Reusch, T.B.H., 2005. How complex life cycle can improve a parasite's sex life. J. Evol. Biol. 18, 1069-1075.

Restif, O., 2009. Evolutionary epidemiology 20 years on: challenges and prospects. Infect. Genet. Evol. 9, 108-123.

Schneider, S., Excoffier, L., 1999. Estimation of demographic parameters from the distribution of pairwise differences when the mutation rates vary among sites: Application to human mitochondrial DNA. Genetics 152, 1079-1089.

Sire, C., Durand, P., Pointier, J.-P., Théron, A., 2001. Genetic diversity of Schistosoma mansoni within and among individual hosts (Rattus rattus): infrapopulation differentiation at microspatial scale. Int. J. Parasitol. 31, 1609-1616.

Slatkin, M., 1991. Inbreeding coefficients and coalescence times. Genet. Res. Camb. 58, 167175.

Steinauer, M.L., Blouin, M.S., Cricione, C.D., 2010. Applying evolutionary genetics to schistosome epidemiology. Infect. Genet. Evol. 10, 433-443.

Templeton, A.R., Crandall, K.A., Sing, C.F., 1992. A cladistic analysis of phenotypic associations with haplotypes inferred from restriction endonuclease mapping and DNA sequence data.III. Cladogram estimation. Genetics 132, 619-633.

Theron, A., Sire, C., Rognon, A., Prugnolle, F., Durand, P., 2004. Molecular ecology of Schistosoma mansoni transmission inferred from the genetic composition of larval and dault infrapopulations within intermediate and definitive hosts. Parasitol. 129, 571578. 
Thompson, J.D., Higgins, D.G., Gibson, T.J., 1994. Clustal W: improving the sensitivity of progressive multiple sequence alignments through sequence wighting, position specific gap penalties and weight matric choice. Nucl. A. Res. 22, 4673-4680.

Traversa, D., Costanzo, F., Iorio, R., Aroch, I., Lavy, E., 2007. Mitochondrial cytochrome c oxidase subunit 1 (cox1) gene sequence of Spirocerca lupi (Nematode, Spirurida): avenues for potential implications. Vet. Parasitol. 146, 263-270.

van der Merwe, L.L., Kirberger, R.M., Clift, S.J., Williams, M., Keller, N., Naidoo, V., 2008. Spirocerca lupi infection in the dog: a review. Vet. J. 176, 294-309.

Weir, B.S., Cockerham, C.C., 1984. Estimating F-statistics for the analysis of population structure. Evolution 38, 1358-1370.

Whitlock, M.C., McCauley, D.E., 1999. Indirect measures of gene flow and migration: $F_{\mathrm{ST}} \neq$ $1 /(4 N m+1)$. Heredity $82,117-125$.

Wu, S.G., Wang, G.T., Xi, B.W., Xiong, F., Liu, T., Nie, P., 2009. Population genetic structure of the parasitic nematode Camallanus cotti inferred from DNA sequences of ITS1 rDNA and the mitochondrial COI gene. Vet. Parasitol. 164, 248-256. 


\section{Tables}

Table 1. The haplotypes of $60 \mathrm{~S}$. lupi individuals sampled from twenty dogs.

\begin{tabular}{|c|c|c|c|}
\hline & \multicolumn{3}{|c|}{ Haplotype } \\
\hline & Worm 1 & Worm 2 & Worm 3 \\
\hline $\operatorname{Dog} 1$ & $1^{\mathrm{a}}$ & 1 & 2 \\
\hline Dog 2 & 1 & 1 & 3 \\
\hline Dog 3 & 1 & $4^{b}$ & 5 \\
\hline $\operatorname{Dog} 4$ & 4 & 5 & 5 \\
\hline Dog 5 & 1 & 6 & 6 \\
\hline Dog 6 & 1 & 5 & 7 \\
\hline $\operatorname{Dog} 7$ & 5 & 8 & 8 \\
\hline $\operatorname{Dog} 8$ & 1 & 5 & 5 \\
\hline Dog 9 & 1 & 5 & 5 \\
\hline Dog 10 & 1 & 4 & 9 \\
\hline Dog 11 & 1 & 1 & 4 \\
\hline Dog 12 & 1 & 1 & 1 \\
\hline Dog 13 & 1 & 1 & 4 \\
\hline Dog 14 & 1 & 4 & 4 \\
\hline Dog 15 & 4 & 5 & 5 \\
\hline Dog 16 & 1 & 10 & 11 \\
\hline Dog 17 & 1 & 1 & 5 \\
\hline Dog 18 & 5 & 5 & 5 \\
\hline Dog 19 & 5 & 5 & 5 \\
\hline Dog 20 & 1 & 5 & 5 \\
\hline
\end{tabular}

${ }^{a}$ Boldface numbers indicates haplotypes from haplogroup 1

${ }^{\mathrm{b}}$ Normal type numbers indicates haplotypes from haplogroup 2. 
Table 2. AMOVA design and results for $\operatorname{cox} 1$.

\begin{tabular}{lccccc}
\hline \multicolumn{1}{l}{ Source of variation } & df & SS & $\begin{array}{c}\text { variance } \\
\text { components }\end{array}$ & $\begin{array}{c}\text { \% of } \\
\text { variation }\end{array}$ & $p$ \\
\hline $\begin{array}{l}\text { Among sides of the } \\
\text { Magaliesberg }\end{array}$ & 1 & 0.933 & -0.149 & -4.61 & 0.883 \\
$\begin{array}{l}\text { Among dogs within } \\
\text { sides of the }\end{array}$ & 18 & 94.167 & 0.922 & 28.45 & 0.009 \\
$\begin{array}{l}\text { Magaliesberg } \\
\text { Within dogs }\end{array}$ & 40 & 98.667 & 2.467 & 76.15 & 0.012 \\
Total & 59 & 193.767 & 3.240 & & \\
\hline
\end{tabular}


Table 3. A summary of studies that quantified between host genetic differentiation of macroparasitic worms.

\begin{tabular}{llll}
\hline Species & Marker & $\begin{array}{l}\text { Between host } \\
F_{S T} \text { or \% of }\end{array}$ & Reference \\
& & genetic variation & \\
\hline Blatticola blattae & RAPD & 0.253 & Jobet et al. \\
Ascaris & mtDNA restriction & 0.206 (humans) & Anderson et al. 1995 \\
& & 0.203 (pigs) & \\
Ascaris suum & isozymes and & 0.09 & Nadler et al. 1995 \\
& RAPD & & \\
Ascaris lumbricoides & microsatellites & 0.081 & Criscione et al. 2007 \\
Schistosoma mansoni & RAPD & 0.08 & Sire et al. 2001 \\
Schistosoma mansoni & RAPD & 0.061 & Theron et al. 2004 \\
Plagioporus shawi & microsatellites & $0-0.01$ & Criscione and Bluoin \\
& & & 2006 \\
Ascaris lumbricoides & microsatellites & 0.005 & Criscione et al. 2010 \\
Trychostrongylus tenius & microsatellites & 0.00 & Johnson et al. 2006 \\
\hline
\end{tabular}




\section{Figures}

Fig. 1. Sample locations in the Pretoria and Centurion area of South Africa (see inset). The thin lines indicate rivers (flowing to the north, is the Apies river and one of its tributaries; in the south, flowing to the east is the Hennops river. The feathered heavy line indicates the eastern tip of Magaliesberg mountain; each circle represents one dog and each third represents one of the three worms sampled per dog. The circle is centred on the locality unless more than one dog was sampled per locality in which case a smaller filled circle indicates the locality. Black colouring indicates that the mitochondrial haplotype falls in the $2^{\text {nd }}$ haplotype group and white colouring refers to haplogroup 1 . Numbers next to the circles indicate the identity of the sampled dog.

Fig. 2. Isoclines of expected $F_{S T}$ values given that a fraction $a$ of infrapopulations were formed by the amalgamation of offspring from two parent infrapopulations and that infrapopulations contain $n$ effective copies of the allele. For mtDNA $n$ is equal to the number of females in the population. The heavy lines bounded by circles connect the observed $F_{S T}=$ 0.272 for mitochondrial DNA to the equivalent expectation for nuclear markers such as microsatellites due to the larger effective population size of nuclear markers.

Fig. 3.The ternary plot of the number of haplotypes per triad of worms from each dog (the axis are number of dogs with three identical worms, two identical or no identical worms). The black circle indicates the observed sample and the colour shading of the other circles correlates with the probability of each possible combination with grey the lowest and browner colours being more likely.

Fig. 4. Gene network for coxl calculated using TCS version 1.21 (Clement et al., 2000). Circle area is correlated with number of individuals of each haplotype (h1 $=22, \mathrm{~h} 2=1, \mathrm{~h} 3=$ $1, \mathrm{~h} 4=8, \mathrm{~h} 5=20, \mathrm{~h} 6=2, \mathrm{~h} 7=1, \mathrm{~h} 8=2, \mathrm{~h} 9=1, \mathrm{~h} 10=1, \mathrm{~h} 11=1)$. Small circles are inferred haplotyes to link sampled haplotypes most parsimoniously. Numbers in circles are the haplotype number and numbers on lines are the sites that are mutated.

Fig. 5. The mismatch distribution of the trimmed (solid) and complete (dashed) sample. 


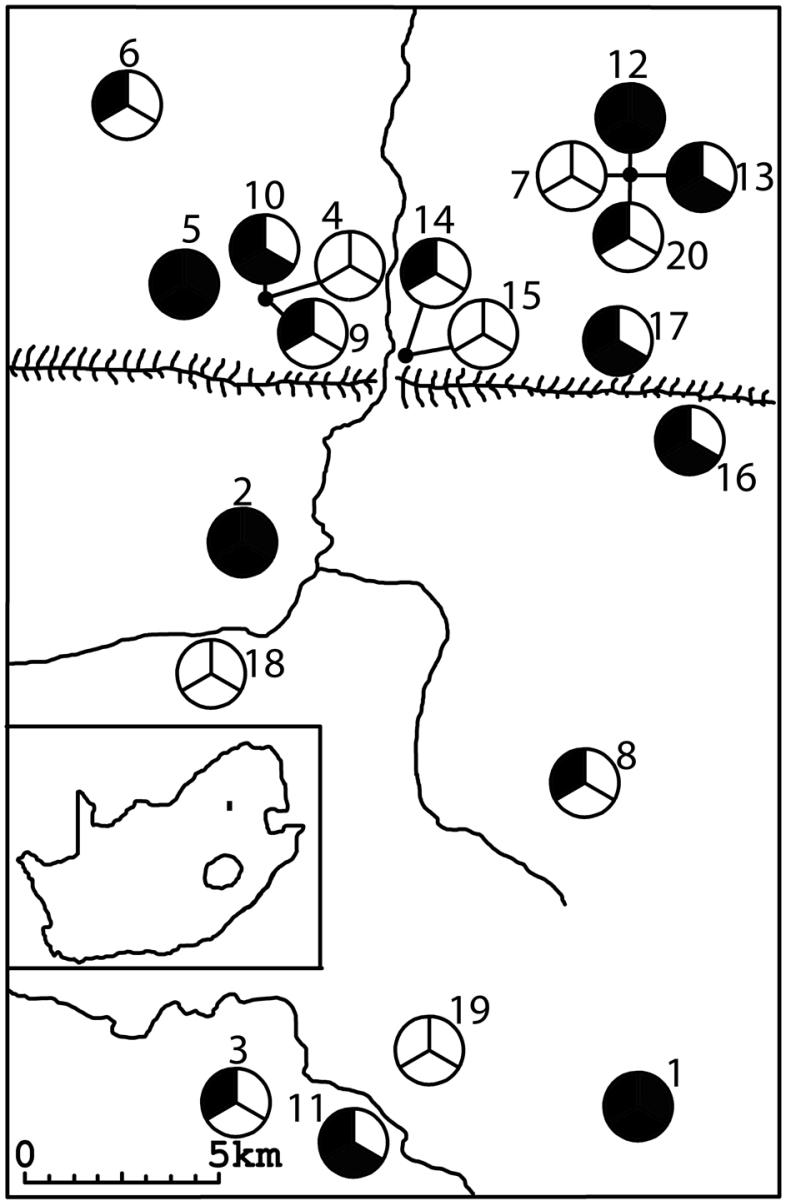




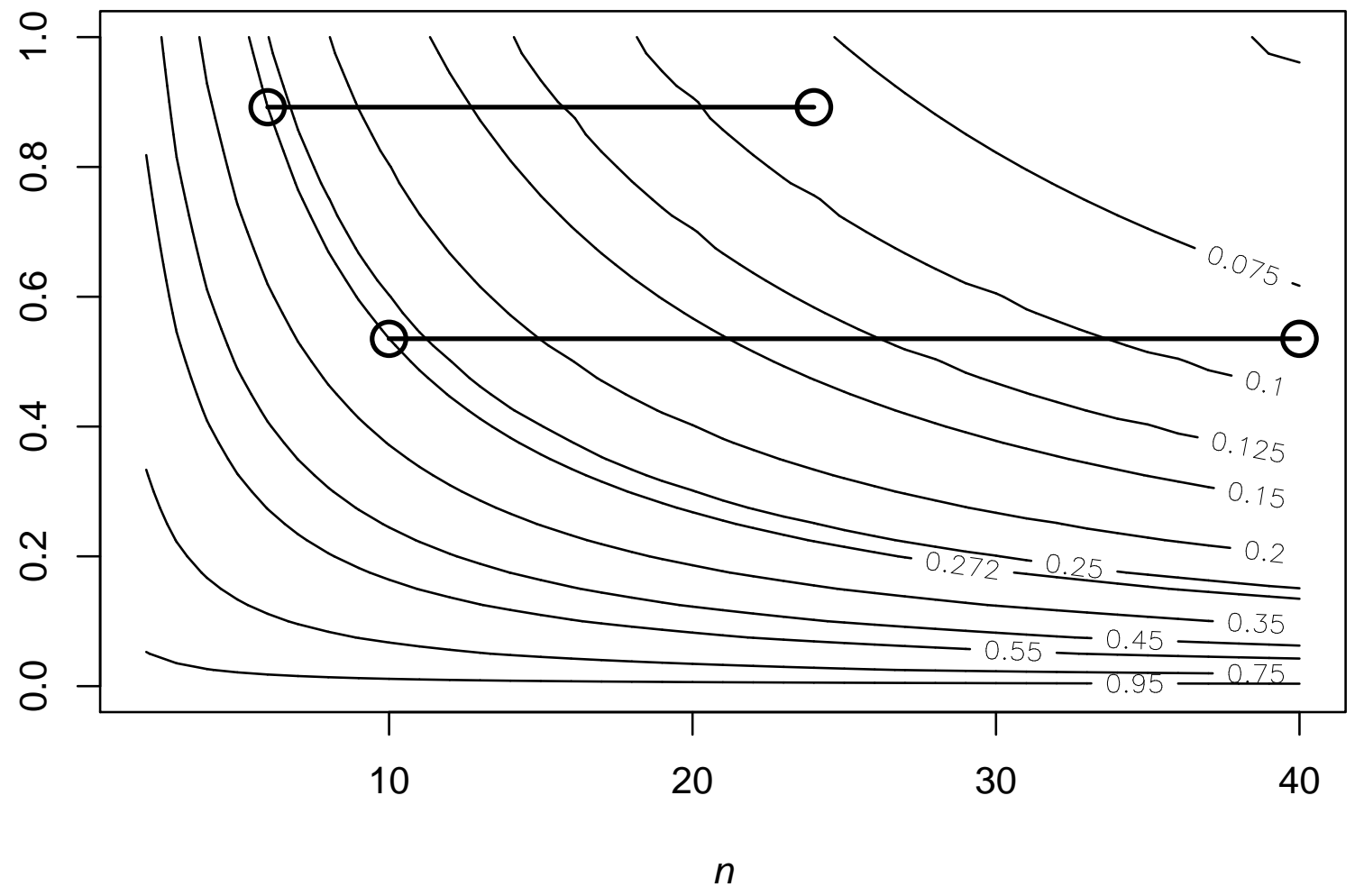


Three

One 


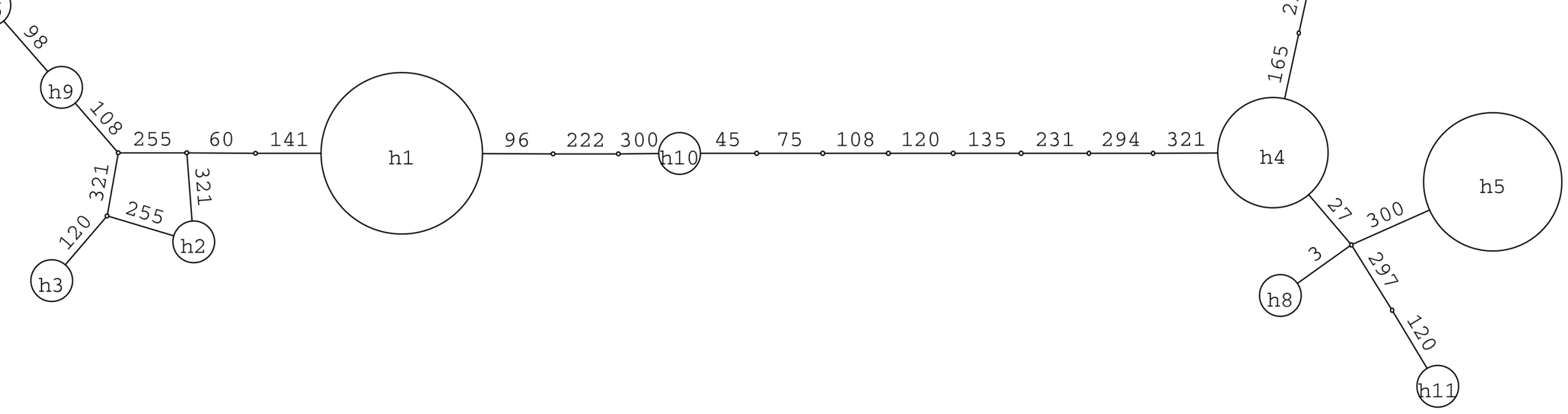




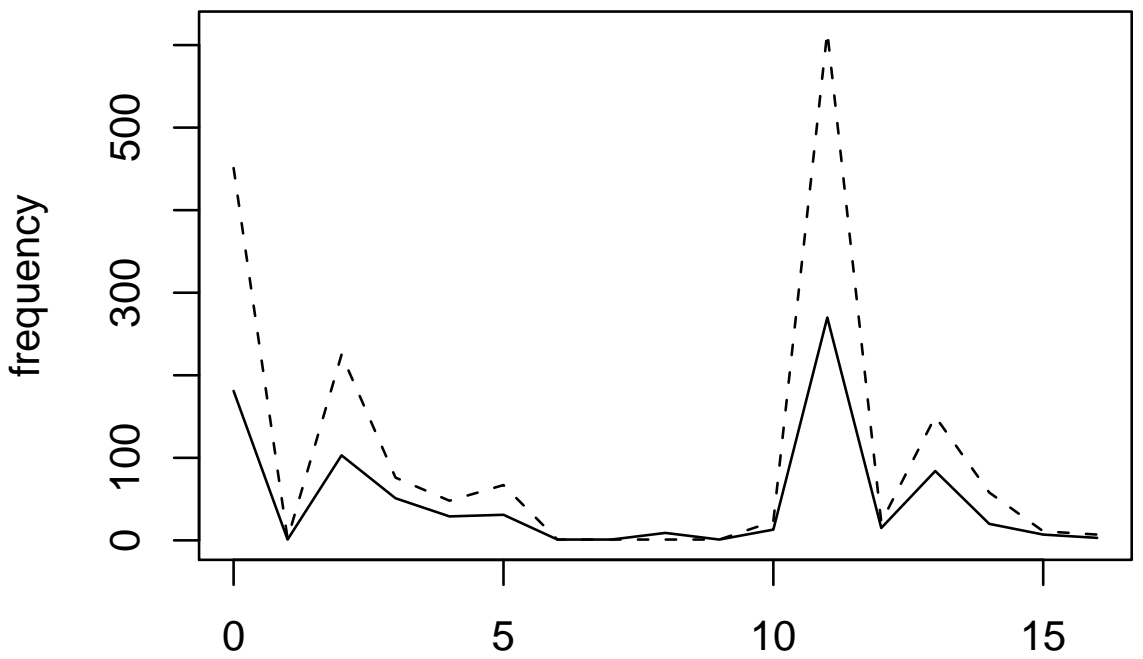

mismatch 